EESTI NSV TEADUSTE AKADEEMIA TOIMETISED. XVII KOIDE

KEEMIA * GEOLOOGIA. 1968,' Nr. 2.

ИЗВЕСТИЯ АКАДЕМИИ НАУК ЭСТОНСКОЙ ССР. ТОМ ХVII

ХИМИЯ * ГЕОЛОГИЯ. 1968, № 2

J. ПААЛЬМЕ, М. ГУБЕРГРИЦ

\title{
МАКРОКИНЕТИКА ФОТОЛИЗА З,4-БЕНЗПИРЕНА В АЭРИРОВАННОМ РАСТВОРЕ ОКТАНА
}

В предыдущей работе авторов [1] охарактеризована кинетика фотохимического превращения 3,4-бензпирена в бензольном растворе при ограниченном доступе воздуха под действием ультрафиолетового облучения. В настоящем сообщении приведены результаты второго этапа аналогичного кинетического исследования этого представителя канцерогенных углеводородов в растворе октана, в тех же условиях фотолиза. Постановка исследования обусловлена необходимостью установить влияние на процесс фотолиза растворителя, резко отличного по своей химической природе от бензола, моделирующего нефтяные углеводороды и обладающего достаточно высокой растворяющей способностью.

\section{Методика}

Основная часть исследования проведена в трех циклах (двадцати сериях) экспериментов. В опытах варьировались: условия мощносгь дозы - от 3 до $8 \cdot 10^{13}$ эв/мл $\cdot$ сек, начальная концентрация бензпирена в растворе - от $3-6$ до $250 \cdot 10^{-6} 2 / \mu \Omega$, длительность экспозиции - от 2 до $24-48$ ч. Методика эксперимента и обработки результатов описана в [1]. В каждой серии дополнительно поставлены холостые опыты с облучением одного лишь растворителя при тех же режимах с целью оценки его поведения в процессе и выполнены дозиметрические определения для нахождения условных значений мощности и величины дозы поглощенной энергии. В работе использованы реактивы марки «хч». Чистота 3,4-бензпирена проверена спектрофотометрически по эталону. Октан подвергнут дополнительной очистке и полной осушке (температура кипения $\left.126^{\circ} \mathrm{C}, n_{D}{ }^{20}=1,398, d=0,703\right)$. Все опыты проводились по-прежнему при температуре $25 \pm 1^{\circ} \mathrm{C}$.

Результаты опытов подвергнуты первичной графической обработке. Во всех случаях экспериментальные точки удовлетворительно укладываются на прямых, описываемых в общем виде уравнением типа

$$
q=a \tau \cdot 10^{-6} \quad 2 / \mu \Omega,
$$

где $q$-- количество превращенного 3,4-бензпирена, $\tau$-- длительность облучения в часах, $a-$ коэффициент пропорциональности, значения которого для каждой серии опытов приведены в последней строке прилагаемой таблишы.

\section{Обсуждение результатов}

Анализ обработанных экспериментальных данных приводит к заключению, что суммарный процесс фотолиза 3,4-бензпирена, так же как и в предыдущей работе, описцвается макрокинетическим уравне- 
нием нулевого порядка. Определенные на этом основании значения постоянной скорости суммарной реакции приведены в таблице в сопоставлении с данными по энергетическому выходу продуктов фотолиза или превращенного вещества и с данными степени превращения за 8 часов облучения.

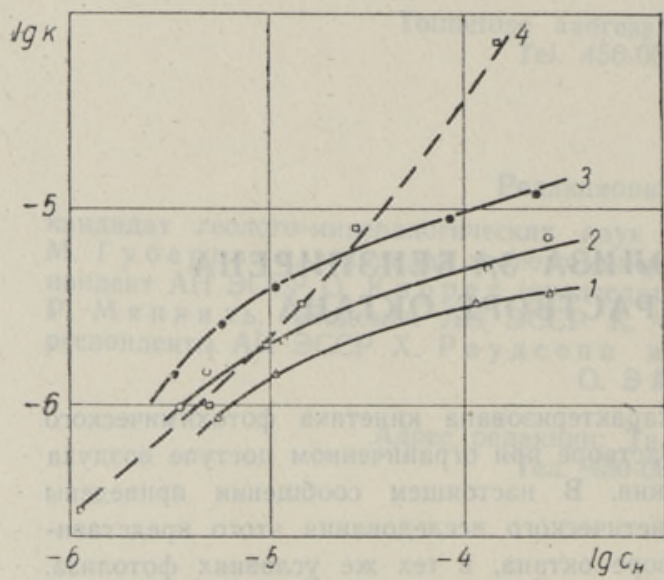

Рис. 1. Зависимость константы скорости суммарной реакции фотохимнческого превращения $(k)$ от начальной концентрации $\left(c_{\text {H }}\right)$ 3,4-бензпирена в аэрированном растворе.

1-3 кривые для аэрированного раствора в октане при помощи поглощенной дозы $3,2-4,5-7,9 \cdot 10^{1}$ эв/мл - сек; 4 - кривая для аэрированного бензольного раствора ["] при мощности дозы $4,5 \cdot 10^{13}$ эв/:.л $\cdot$ сек. График дан в логарифмической системе координат.

Обсуждение полученных данных позволяет сделать заключение, что и в настоящей снстеме скорость суммарной реакции закономерно возрастает с повышением начальной концентрации $\left(c_{\text {H }}\right)$ бензпирена в растворе. Это явление интерпретировано графически на рис. 1. Семейство параллельных кривых, построенных для трех значений мощности дозы, по своему характеру (тенденция к затуханию) существенно отличается от кривой из предыдущей работы для фотолиза в бензольном растворе (кривая 4, рис. 1).

На рис. 2 охарактеризована взаимосвязь энергетического выхода превращенного 3,4-бензпирена и начальной концентрации его в растворе. Для построения кривой жспользованы энспериментальные данные из всех циклов опыта. Все точки хорошо укладываются на одной кривой. Это показывает, что изменение мощности дозы в изученных пределах не влияет на механизм процесса. Указанное обстоятельство, а также установленная нами тенденция к спижению степени превращения исходного вещества за данный отрезок

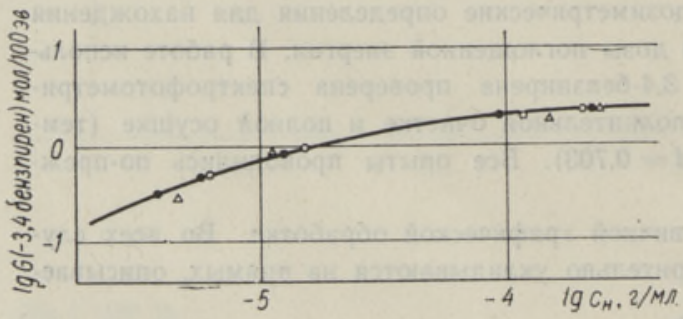

Рис. 2. Условный энергетический выход $($ мол/100 эв) продуктов фотолиза 3,4-бензпирена как функция начальной концентрации последнего в растворе октана в диапазоне мощности поглощенной дозы $3,2-$ $7,9 \cdot 10^{13}$ эв/м $\cdot$ сек.

времени с повышением его начальной концентрации (см. таблицу) и ограниченная абсолютная величина энергетического выхода продуктов фотолиза приводят нас к заключению об отсутствии цепных реакций в суммарном процессе в условиях изучаемсй системы, в противоположность выводам, сделанным на основе результатов предыдущего исследования (бензольный раствор). Некоторый же рост скорости реакции и величины энергетического выхода продуктов превращения при повышении концентрации канцерогена в растворе можно объяснить низким содержанием его в облучаемой смеси и неизбежным расходованием части поглощенной энергии на фотохимическое превращение молекул растворителя. 


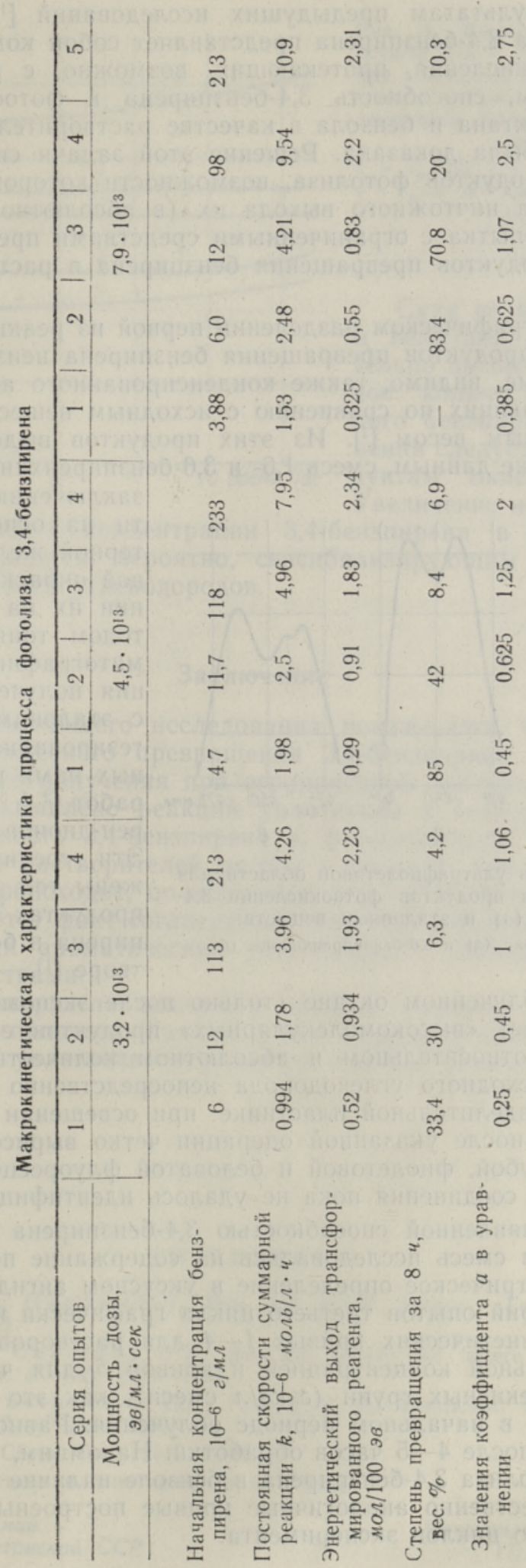


Судя по результатам предыдущих исследований $[1,2]$, суммарный процесс фотолиза 3,4-бензпирена представляет собой комплекс реакций уплотнения и окисления, протекающих, возможно, с различной скоростью. Впрочем, способность 3,4-бензпирена к фотоокислению при использовании октана и бензола в качестве растворителей экспериментально еще не была доказана. Решение этой задачи связано с изучением состава продуктов фотолиза, возможности которого чрезвычайно ограничены из-за ничтожноғо выхода их (в абсолютном выражении). Bсе же такая попытка с ограниченными средствами предпринята нами в отношении продуктов превращения бензпирена в растворе и чистого растворителя.

При хроматографическом разделении первой из реакционных смесей обнаружен ряд продуктов превращения бензпирена неизвестного (судя по хроматограмме, видимо, также конденсированного ароматического) строения, обладающих по сравнению с исходным веществом повышенным молекулярным весом [1]. Из этих продуктов выделена, судя по приведенным ниже данным, смесь 1,6- и 3,6-бензпиренхинонов. К такому
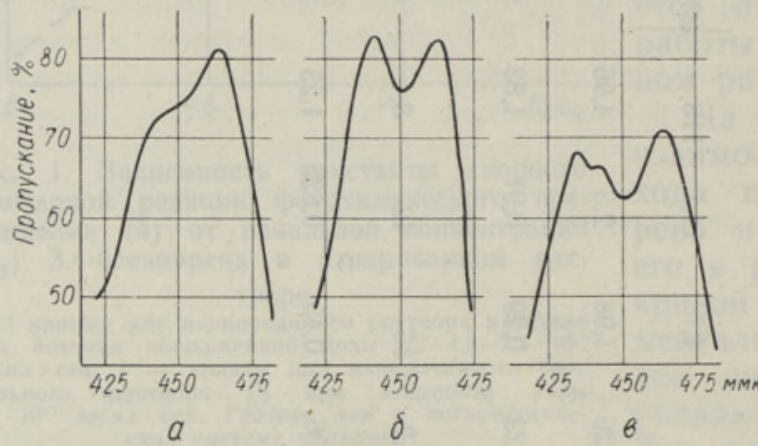

Рис. 3. Спектры в ультрафиолетовой области для выделенной смеси продуктов фотоокисления 3,4б́ензпирена (в) и эталонных веществ:

1,6-бензпиренхинона (a) и 3,6-бензпиренхинона (6), заключению можно прийти на основании характерной желтой и оранжевої окраски при разделении их на пластинке метсдом тонкослойной хроматографии и сопоставления полученных спектров с эталонами [3] для синтезированных и разделенных нами по методике из работ [2, 4, 5] бензальфапирен-дионов (см. рис. 3). Эти соединения обнаружены тем же способом в продуктах фотолиза бензпирена в бензольном растворе [1].

Наличие в облученном октане (только после экспозиции в течение 24 часов и более) «высокомолекулярных» продуктов его фотолиза в незначительном относительном и абсолютном количестве установлено выпариванием исходного углеводорода непосредственно из слоя окиси алюминия на разделительной пластинке: при освещении ее ультрафиолєтовым светом после указанной операции четко вырисовываются три голосы - с голубой, фиолетовой и беловатой флуоресценцией. Входящие в их состав соединения пока не удалось идентифицировать.

В связи с выявленной способнослью 3,4-бензпирена к фотоокислению реакционная смесь исследовалась на содержание перекисных соединений (йодометрическое определение в уксусном ангидриде). Результаты четырех серий опытов третьего цикла графически изображены на рис. 4 в виде кинетических кривых $1-4$ для растворов бензпирена с различной начальной концентрацией и кривой 5 для чистого октана. Содержание перекисных групп (әэкв/л смеси), как это видно из графика, возрастает в начальном периоде облучения. Равновесное состояние достигается после $4-5$ часов обработки. Напомним, что установить в продуктах фотолиза 3,4-бензпирена в бензоле наличие перекисей нам не удалось. Качественно аналогичные кривые построены и по данным первого и второго циклов эксперимента. 


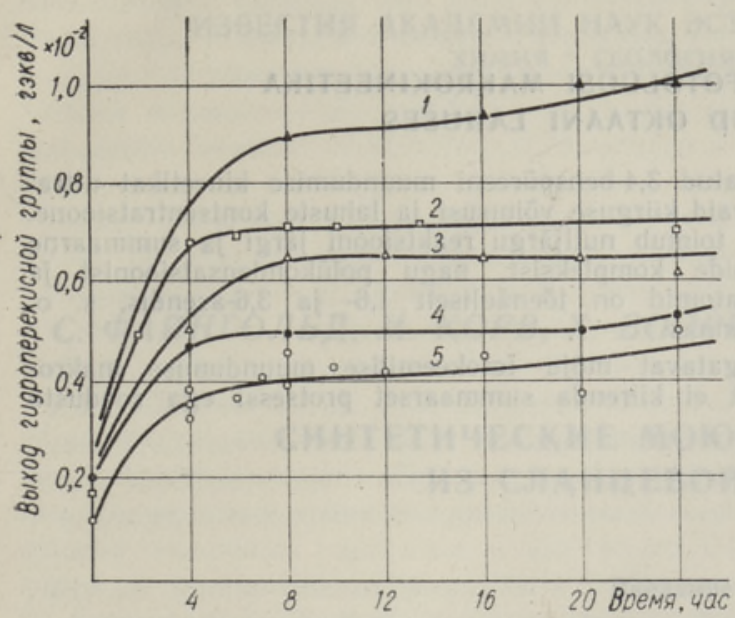

Рис. 4. Выход гидроперекисной группы $\left(10^{-2}\right.$ гэкв/ $\left.\Omega\right)$ во времени при мощности дозы $7,9 \cdot 10^{13} э в / \mathrm{M}$. - сек н различной начальной концентрации 3,4-бензпирена в растворе.

$1-c_{\mathrm{H}}=213 ; 2-c_{\mathrm{H}}=98 ; 3-c_{\mathrm{H}}=12$; $4-c_{\text {н }}=6 \cdot 10^{-5}$ a/M $; 5-4$ чнстый октан $\left(c_{\mathrm{H}}=0\right)$.

Судя по кривой 5 рис. 4 и величине выхода, значительно превышающей исходное количество растворенного бензпирена, эти соединения следуст отнести к продуктам окисления октана. Увеличение же выхода перекисей с повышением концентрации 3,4-бензпирена в растворе (кривые 1-4) объясняется, вероятно, сенсибилизирующим действием добавки ароматических углеводородов.

\section{Заключение}

Результаты настоящего исследования показывают, что суммарный процесс фотохимического превращения 3,4-бензпирена под действием ультрафиолетового облучения при ограниченном доступе воздуха представляет собой комплекс реакций уплотнения и окисления. Впервые доказана способность 3,4-бензпирена к фотоокислению при использовании в качестве растворителей октана и бензола; при этом размещение кислорода происходит, по-видимому, в $L$ - и $M$-областях реагирующей молекулы. Этот факт согласуется с теорией и практикой окисления конденсированных ароматических углеводородов, обладающих канцерогенными свойствами.

Природа растворителя оказывает заметное влияние на макрокинетику суммарного процесса. Участие в нем октана не способствует ускорению процессов уплотнения и развитию цепных реакций.

Л ИТ Р А Т У Р А

1. Губергриц М., Па альме Л., Изв. АН ЭССР. Химия * Геология, 16, № 1 , $32-36$ (1967).

2. Antonello C., Carlassare F., Atti Ist. veneto Sci lettere ed arte, Cl. Sci mat. e natur, $\mathrm{n}^{\circ} 122,1963-1964$.

3. Milazzo G., Casinovi C. G., Ciasca M. A., Rendiconti instituto superiore die sanita, 13, $\mathrm{n}^{\circ} 112(1960)$.

4. Cook J. W., J. Chem. Soc., 1112 (1950).

5. Ве т тиг К., Гигиена и санитария, № 12 (1964).

Институт химии

Академии наук Эстонской ССР
Поступнла в редакцию $17 / \mathrm{X} 1967$ 


\section{PAALME, M. GUBERGRITS}

\section{3,4-BENSPUREENI FOTOLUUSI MAKROKINEETIKA AEREERITUD OKTAANI LAHUSES}

Uuriti aereeritud oktaanis lahustatud 3,4-benspüreeni muundumise kineetikat ultraviolettkiirguse mõjul, kasutades erinevaid kiirguse võimsusi ja lahuste kontsentratsioone. Leiti, et 3,4-benspüreeni muundumine toimub nulljärgu reaktsiooni järgi ja summaarne protsess ilmselt koosneb reaktsioonide kompleksist, nagu polükondensatsioonist ja hapendumisest, kusjuures hapniku aatomid on tõenäoliselt 1,6- ja 3,6-asendis, s. 0 . kantserogeeni molekuli M- ja L-piirkonnas.

Lahustaja loomus avaldab märgatavat mõju fotokeemilise muundumise makrokineetikale, kusjuures oktaani osavõtt ei kiirenda summaarset protsessi ega soodusta. ahelreaktsioonide kulgu.

\section{PAALME, M. GOUBERGRITS}

\section{LA CINETIQUE DU PHOTOLYSE DU BENZO $(3,4)$ PYRENE EN SOLUTION D'OCTANE AERE}

L'article concerne les résultats d'une étude sur la cinétique du photolyse sous l'irradiation ultraviolette de la solution du benzo $(3,4)$ pyrène en octane partiellement aéré. L'intensité de la dose absorbée se varie en limites dès 3 à $8 \cdot 10^{13} \mathrm{eV} / \mathrm{ml} \cdot \mathrm{sec}$, la durée d'exposition - dès 2 à $24-48 \mathrm{~h}$, la concentration initiale du cancérogène dès $3-6$ à $250 \cdot 10^{-6} \mathrm{~g} / \mathrm{ml}$ (sous la température $25 \pm 1^{\circ} \mathrm{C}$ ).

La réaction intégrale étant d'ordre zéro, tous les indices macrocinétiques sont établis en fonction de paramètres variables du procédé. La transformation photolytique du benzopyrène est considérée comme un complexe des réactions d'origine diverse, pàrticulièrment - de la condensation et de l'oxydation. L'adjonction d'oxygène a lieu plutôt dans les regions $L$ et $M$ des molécules du cancérogène. Cela se prouve par la formation d'un mélange des 1,6 et 3,6-benzo $(3,4)$ pyrène-quinones comme la partie: intégrante des produits formés du photolyse.

L'origine chimique du solvant a une influence considérable sur la macrocinétique de la dégradation. La participation d'octane en réactions de la condensation (ce composé est plutôt le sujet d'oxydation) semble être négligeable. 